\title{
Bayesian approach for analysis of time-to-event data in plant biology
}

\author{
Jan F. Humplík ${ }^{1 *} \mathbb{D}$, Jakub Dostál², Lydia Ugena', Lukáš Spíchal ${ }^{1}$, Nuria De Diego ${ }^{1}$, Ondřej Vencálek² \\ and Tomáš Fürst ${ }^{1,2}$
}

\begin{abstract}
Background: Plants, like all living organisms, metamorphose their bodies during their lifetime. All the developmental and growth events in a plant's life are connected to specific points in time, be it seed germination, seedling emergence, the appearance of the first leaf, heading, flowering, fruit ripening, wilting, or death. The onset of automated phenotyping methods has brought an explosion of such time-to-event data. Unfortunately, it has not been matched by an explosion of adequate data analysis methods.
\end{abstract}

Results and discussion: In this paper, we introduce the Bayesian approach towards time-to-event data in plant biology. As a model example, we use seedling emergence data of maize under control and stress conditions but the Bayesian approach is suitable for any time-to-event data (see the examples above). In the proposed framework, we are able to answer key questions regarding plant emergence such as these: (1) Do seedlings treated with compound A emerge earlier than the control seedlings? (2) What is the probability of compound A increasing seedling emergence by at least 5 percent?

Conclusion: Proper data analysis is a fundamental task of general interest in life sciences. Here, we present a novel method for the analysis of time-to-event data which is applicable to many plant developmental parameters measured in field or in laboratory conditions. In contrast to recent and classical approaches, our Bayesian computational method properly handles uncertainty in time-to-event data and it is capable to reliably answer questions that are difficult to address by classical methods.

Keywords: Data analysis, Bayesian inference, Plant development, Statistics, Uncertainty, Time-to-event data, Survival analysis, Plant phenotyping

\section{Background}

Reliable and robust tools for the analysis of highthroughput experimental data are reported to be the current bottleneck in plant phenotyping, breeding, and agricultural research [1]. Despite the current replication crisis and the dawning of "post $\mathrm{p}<0.05$ era", this task is still not reflected enough in plant biology $[2,3]$. There is overwhelming literature on how to analyze time-to-event

\footnotetext{
*Correspondence: jan.humplik@upol.cz

${ }^{1}$ Department of Chemical Biology and Genetics, Centre of the Region Haná for Biotechnological and Agricultural Research, Faculty of Science, Palacký University, Šlechtitelů 27, 78371 Olomouc, Czech Republic Full list of author information is available at the end of the article
}

data, mostly based on seed germination datasets. Ranal et al. [4] report that the number and diversity of mathematical expressions measuring various aspects of the germination process make any comparison difficult. They list a table of 19 parameters that have been used in the domain of germination assays only.

Most of the statistical treatment proceeds as follows [4, 5]: suppose we want to compare the median time to germination $\left(t_{50}\right)$ in two seed populations, $A$ and $B$. We prepare five trays of seeds from population A (100 seeds per tray) and five trays of seeds from population B (100 seeds per tray). We collect the data and fit a parametric growth model (e.g., a logistic curve) to the data. Thus, we obtain 
five values for $t_{50}$ for the seeds from population $\mathrm{A}$ and five values of $t_{50}$ for the seeds from population $\mathrm{B}$. Then we perform a $t$-test to compare whether the $t_{50}$ value for the seeds from population A differs from the corresponding value for the seeds from population B. Notice that this procedure is independent of the number of seeds in each tray! The test has the same power, irrespective of whether each tray contains ten seeds or a million seeds. This is clearly inadequate, and yet this type of data evaluation is used more often than not [5].

One way out of this problem is to use the confidence intervals of the parameter estimates from the nonlinear regression [6]. It is clear that the width of the confidence intervals reflects the number of seeds in the tray. However, growth curve fitting by means of nonlinear regression is not appropriate for time-to-event data because the regression models assume a different underlying mechanism (see the Additional file 1 for a full explanation). Several authors noticed the inadequacy of nonlinear regression for growth models, most notably [7-10]. McNair et al. [7] correctly report that confidence intervals, a hypothesis test for parameter values, and a comparison of parameters for two or more groups that are provided by standard statistical software must be disregarded.

As has been reported previously, the correct approach is to embrace survival analysis methods for time-to-event data $[7,8,10]$. However, even survival analysis comes in two flavors: Classical (frequentist) and Bayesian. We offer a novel, general-purpose, easy-to-understand and flexible Bayesian tool to analyze any type of time-to-event data and to answer the most common scientific questions regarding this type of data.

Over the last century, survival analysis has provided us with many tools [11-13] to analyze time-to-event data (see also $[7,8]$ for a review). Most of them can readily be used in plant biology to analyze emergence or germination assays. In our case, the primary quantity of interest is the emergence function $F(t)$, which denotes the probability that a plant will emerge before time $t$. In practice, we often perform experiments to quantify the effect of the stressor (or a stimulant). To this end, a regression model must be used. The Cox proportional hazards (PH) model is the obvious candidate and the one that is used almost exclusively but it has a very strong assumption: it assumes that the effect of the predictor (e.g., a stressor) is constant in time. It is very likely that this assumption does not hold in many biological settings [14], especially in seedling emergence, which is connected with photomorphogenesis, one of the most dramatic periods in the life of plants. Moreover, we point out that none of the classical methods used for survival analysis is able to answer questions of type 2 above. To do that, we have to turn to Bayesian methods.

Lately, various machine learning (ML) methods have emerged in all fields that collect large amounts of data. It is natural to ask whether ML methods may be useful for answering the questions in the abstract. However, state-of-the-art ML methods deal with regression or classification (which are usually further used for prediction), but they have no tools to address the uncertainty of the result. Without the accompanying uncertainty, one cannot answer how probable it is that the classification offered by a ML tool is correct. From the point of view of statistics, ML method provide sophisticated but only point estimates. For most of the methods, estimates of variability are not yet available. Thus ML methods address a fundamentally different question than this article.

\section{Results}

We illustrate the approach through a maize emergence experiment (see "Methods" for details). Let us first concentrate on a single tray of 110 seeds to introduce the Bayesian framework. The tray was observed every $2 \mathrm{~h}$ for 12 consecutive days. The number of plants that did not emerge at all is denoted by $M$. For each plant that emerged, we recorded during which 2-h interval it happened. Thus, for each interval $\left[t_{i} t_{i+1}\right]$, we know the number $N_{i}$ of plants that emerged during this interval. Here $t_{0}=0, t_{2}=2, t_{3}=4, \ldots, t_{144}=288$ because $288 \mathrm{~h}$ correspond to 12 days. Obviously

$$
\sum_{i=0}^{T-1} N_{i}=110-M,
$$

where $T=144$ denotes the number of 2 -h intervals during the 12 days of the experiment. Let us denote the probability that a plant emerges before time $t$ by $F(p, t)$, where by $p$ we stress that the function depends on some parameters. If we fix the value of the parameters $p$, it is easy to write down the probability of the observed data. This probability (called the likelihood function) is a product of 110 terms because we assume that the emergence times of the individual plants are independent. For each plant that did not emerge at all, we multiply the likelihood by the term $\left[1-F\left(p, t_{T}\right)\right]$, which gives the probability that a plant will not emerge before the end of the experiment. If a plant emerged between $t_{i}$ and $t_{i+1}$, we multiply the likelihood by the term $\left[F\left(p, t_{i+1}\right)-F\left(p, t_{j}\right)\right]$, which gives the probability that a plant will emerge before $t_{i+1}$ but not before $t_{i}$. Altogether, the likelihood function can be written as 


$$
L(p)=\left[1-F\left(p, t_{T}\right)\right]^{M} \prod_{i=0}^{T-1}\left[F\left(p, t_{i+1}\right)-F\left(p, t_{i}\right)\right]^{N_{i}}
$$

Note that the product runs over all the intervals, not all the plants that have emerged. Any interval with $N_{i}=0$ will produce a unity and thus not alter the value of the product. Formula (1) is interpreted as the probability of the observed data, given the values of the parameters. Let us now change the point of view: consider the set of all possible values of the parameters. To each point in this parameter space, formula (1) assigns the probability of the observed data given these particular values of the parameters. Thus, formula (1) can be viewed as a function over the parameter space. Low values of $L$ indicate values of parameters that are unlikely to produce the observed data. High values of $L$ reveal the parameters which are likely to produce the observed data.

Here, a minor departure from standard parametric Bayesian models is needed. Let us assume that there are, in fact, two types of seedlings: those that would eventually emerge if we observed them for long enough, and those that would never emerge. Let us use alpha to denote the probability that a seedling will emerge eventually (i.e., (1alpha) is the probability that it will never emerge). Then the probability that a seedling will emerge before time $t$ can be written as

$$
\begin{aligned}
& \operatorname{prob}(\text { seedling emerges before } \mathrm{t}) \\
& =\operatorname{prob}(\text { seedling emerges before } \mathrm{t} \mid \text { it is dead }) \operatorname{prob}(\text { it is dead }) \\
& \quad+\operatorname{prob}(\text { seedling emerges before } \mathrm{t} \mid \text { it is alive }) \operatorname{prob}(\text { it is alive }) \\
& =0 *(1-\text { alpha })+\mathrm{F}(\mathrm{t}) * \text { alpha }
\end{aligned}
$$

Here $F(t)$ again stands for a standard parametric growth model, e.g., the Gompertz distribution. The likelihood function (1) has to be altered accordingly: for each seedling which was not observed until the end of the experiment, we multiply the likelihood by the term

$$
(1-\text { alpha })+\text { alpha } *\left(1-F\left(p, t_{T}\right)\right)
$$

This captures the probability that we did not see the plant emerge either because it was dead (the term (1alpha)) or because it was alive but would have emerged after the end of the experiment (the term alpha* (1 - $\left.\left.F\left(p, t_{T}\right)\right)\right)$. If a plant emerged between $t_{i}$ and $t_{i+1}$, we multiply the likelihood by the term

$$
\text { alpha } *\left[F\left(p, t_{i+1}\right)-F\left(p, t_{i}\right)\right],
$$

which gives the probability that the plant was alive and emerged before $t_{i+1}$ but not before $t_{i}$. Altogether, the likelihood function becomes

$$
L(p)=\left[1-\alpha F\left(p, t_{T}\right)\right]^{M} \prod_{i=0}^{T-1}\left[\alpha F\left(p, t_{i+1}\right)-\alpha F\left(p, t_{i}\right)\right]^{N_{i}}
$$

We will be ready to answer questions 1 and 2 once we understand how to treat the uncertainty of the parameter estimates provided by (2). Let us use the Gompertz model, which assumes

$$
F(t)=\exp [-\exp (B(t-C))]
$$

There are three parameters to be estimated: the emergence yield alpha, emergence uniformity $B$, and emergence half-time $C$. Bayesian inference makes us spell out what we think about these parameters before we perform the experiment. Thus, each of the parameters is assigned a prior distribution which quantifies our degree of belief in the parameter's values. Since alpha must lie in the interval $[0,1]$, we assume its prior distribution to be the beta distribution, e.g., with the parameters $[1,9] . B$ and $C$ are assumed to have a Gaussian prior with $\mathrm{mu}_{\mathrm{B}}=2$, $\operatorname{std}_{B}=0.5$, and $\operatorname{mu}_{C}=5, \operatorname{std}_{C}=2$. Figure 1a shows a hundred samples of emergence curves drawn from this prior distribution.

After the data are observed, the prior distribution is updated according to Bayes's Law, which states that

$$
\begin{aligned}
& \text { Posterior }(\mathrm{p})= \operatorname{prior}(\mathrm{p}) * \text { likelihood }(\mathrm{p}, \text { data }) / \\
& \text { evidence }(\text { data }) .
\end{aligned}
$$

The posterior represents the distribution of our degree of belief in the values of the parameters after observing the experimental data. The posterior directly quantifies the uncertainty of the values of the parameters together with the correlation among the parameters. Once the posterior is computed, all questions of the type 1 and 2 can easily be answered. The likelihood function in Eq. (3) has already been introduced and it is given by Eq. (2). The evidence in the denominator of (3) represents a normalization factor that is independent of the parameters and which need not be computed. Omitting the denominator, one computes an un-normalized posterior which is then rescaled to represent a probability density function (i.e., it is rescaled to a unit integral over the parameter space).

Let us now use Eq. (3) together with the likelihood function given by (2) to obtain the posterior. Figure $1 \mathrm{~b}$ shows 100 emergence curves with parameters drawn from the posterior given by (3). We can observe that our vague initial belief in the shape of the emergence curve (shown in Fig. 1) was updated by the data and resulted in a fairly precise estimate of the emergence curve. 
a

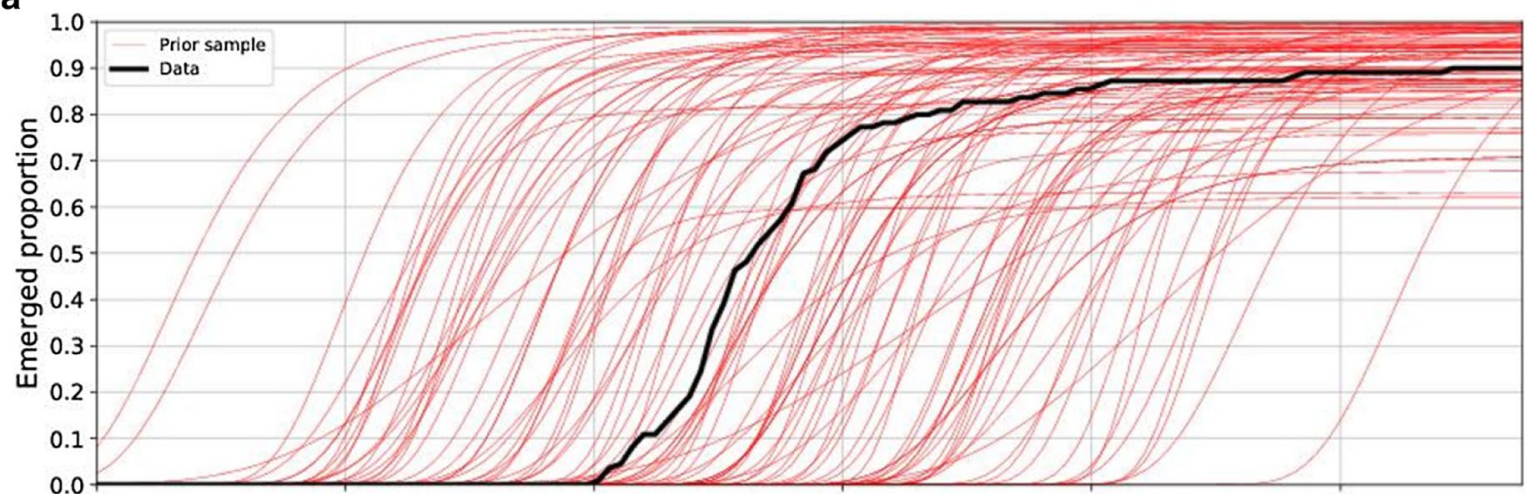

b

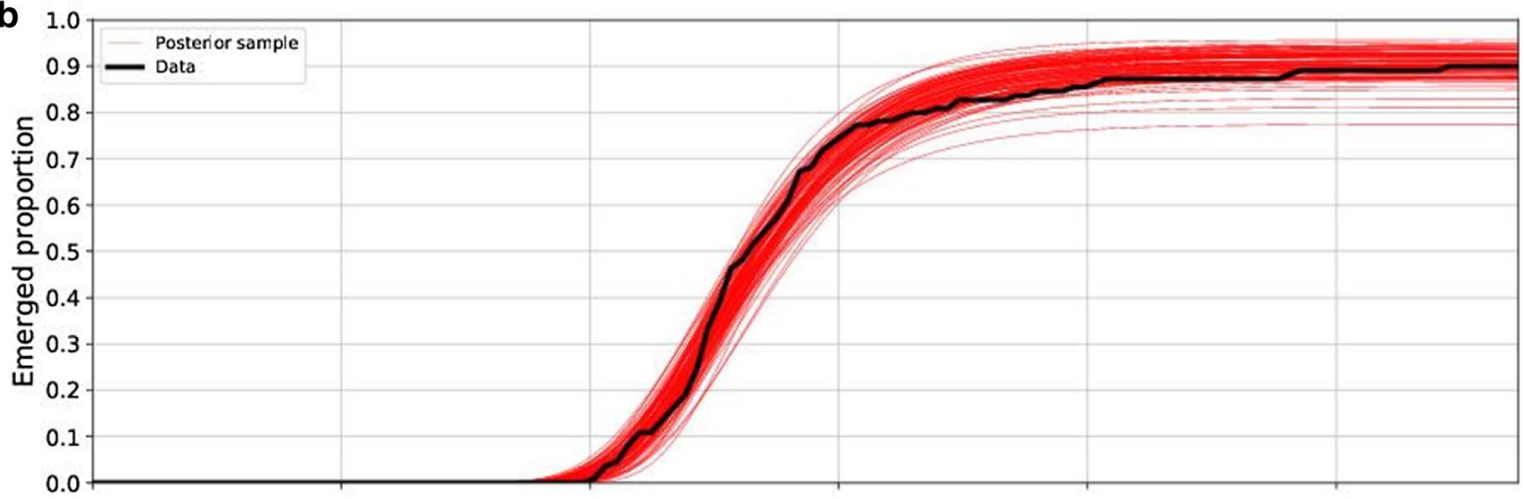

C

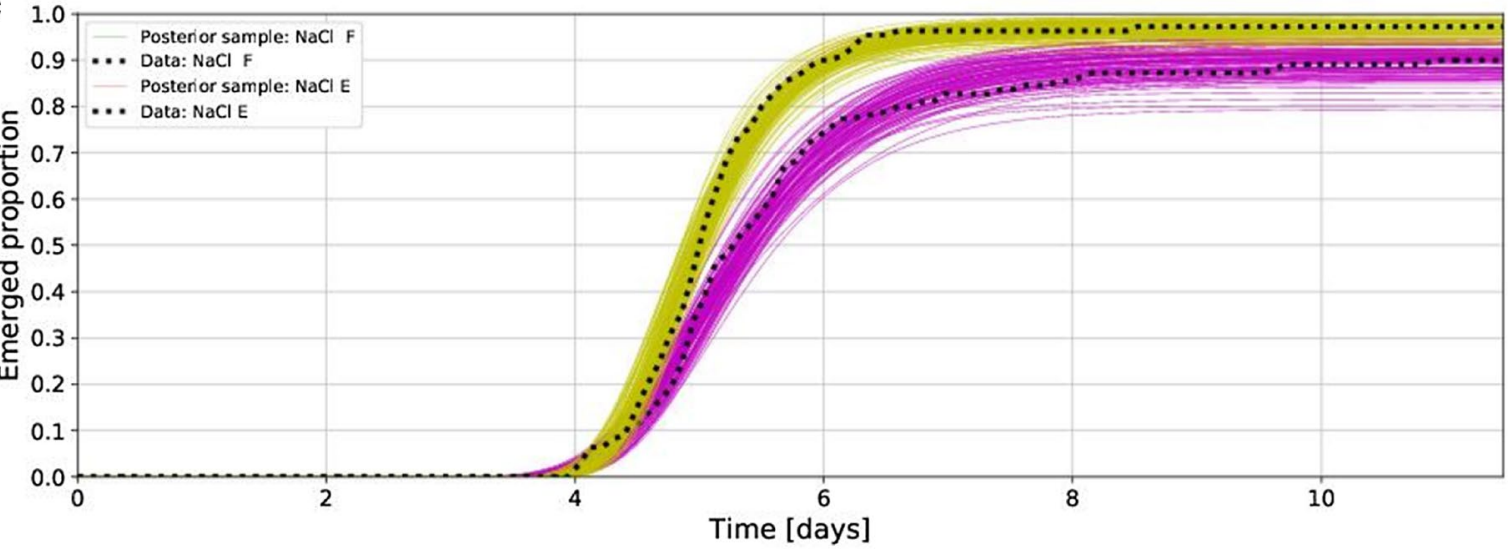

Fig. 1 a Hundred samples of the possible emergence curves drawn from the prior distribution of the parameters. The data are shown in black but unused at this stage. Note that all the curves are increasing — this is a prior knowledge built into the model. $\mathbf{b}$ One hundred samples of the possible emergence curves drawn from the posterior. The difference between $\mathbf{a}$ and $\mathbf{b}$ shows how much our belief about the parameters of the emergence curve changed as a result of observing the data (shown in black). c Comparison of two emergence curves by means of sampling from the posterior. One hundred samples shown for each variant. See "Methods" for a description of the experimental design

\section{Discussion}

Let us now return to questions 1 and 2 from the introduction to illustrate practical application of Bayesian approach.

1. Do seedlings treated with compound A emerge earlier than the control seedlings?
We have two sets of data, one for the control seeds and one for the primed seeds. We observe the emergence times and find the posterior distribution for the parameter $C$ in both cases. Then we draw, e.g., a thousand samples of $C$ from the posterior of the control $\left(C^{1}{ }_{1}-C^{1}{ }_{1000}\right)$ and a thousand samples of $C$ from the posterior of the treated seeds $\left(C^{2}{ }_{1}-C^{2}{ }_{1000}\right)$. The number of times $C^{1}$ is less than $C^{2}$ provides the esti- 
mate of the probability of seedlings from the primed seeds emerging earlier than seedlings from the control seeds. Figure 1c shows 100 emergence curves for both sets of data (data provided in the template file for the online evaluation web application described in the Additional file 1). The probability that $C^{1}$ is less than $C^{2}$ was estimated to be $p=0.01$, i.e., there is a more than $99 \%$ probability that seedlings from population A will emerge earlier than seedlings from the control population in the given conditions.

2. What is the probability of compound E increasing seedling emergence in salinity $(\mathrm{NaCl})$ by at least 5 percent with respect to compound F?

Here we need to estimate the probability that the parameter alpha is at least 5 percent higher in the seeds primed with $E$ than in the seeds primed with $\mathrm{F}$, sown both into $\mathrm{NaCl}$ containing substrate (see Fig. 1). We draw, e.g., a thousand samples (alpha ${ }_{1}^{1}$ - alpha ${ }_{1000}$ ) from the posterior of the group F and a thousand samples $\left(\right.$ alpha $^{2}{ }_{1}-$ alpha $\left.^{2}{ }_{1000}\right)$ from the posterior of the group E. We compute the number of times alpha $a^{2} \geq 1.05 *$ alpha ${ }^{1}$. This shows that there is a 73 percent probability that compound $\mathrm{E}$ increases seedling emergence by at least 5 percent with respect to compound $\mathrm{F}$ in the given conditions.

It is difficult to imagine a relevant question which could not be answered in this straightforward way once the posterior is computed. The posterior distribution of the parameters can be used to compare a certain trait across the whole experiment. For example, Fig. 2b shows the plot of the posteriors for emergence half-time (parameter $C$ ) across the entire population that was tested. Sampling methods are needed to work with the posteriors but they are readily available nowadays in various software tools. We performed the sampling in Python $[15,16]$. A free version of the simulation software is available at http://www.bayes4plan ts.com and described in the Additional file 1. The full code is freely available here.



b

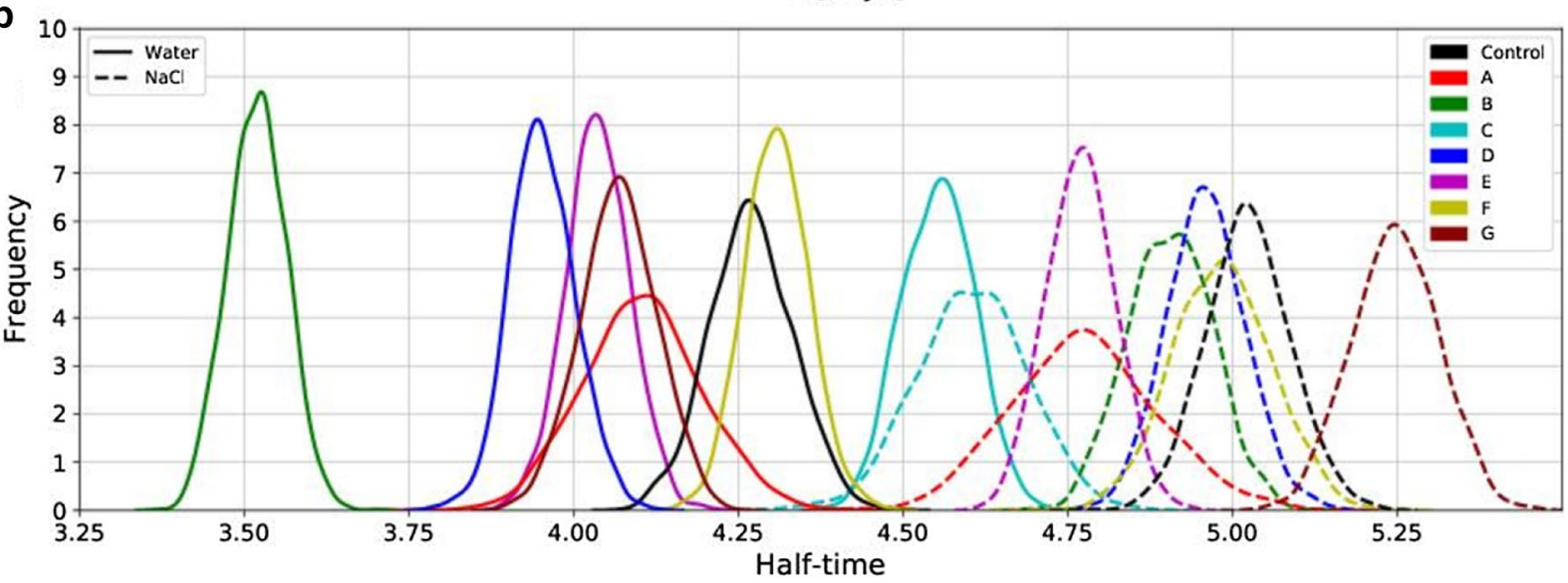

Fig. 2 a Detail of empirical emergence curves for seeds primed with all the compounds that were tested. The significance of the differences among the curves are not obvious. b Posterior distributions for emergence half-time (parameter $\mathrm{C}$ in Table 1) across the entire population that was tested. The differences among the various compounds are clearly visible and can be quantified 
Table 1 Summary of the mean value (expectation) of the posterior distribution of all three parameters for all the compounds that were tested

\begin{tabular}{llll}
\hline & Alpha & B & C \\
\hline Control water & 0.93 & 1.65 & 4.27 \\
Control NaCl & 0.96 & 1.54 & 5.03 \\
A water & 0.98 & 1.19 & 4.11 \\
A NaCl & 0.98 & 0.93 & 4.78 \\
B water & 0.97 & 2.33 & 3.52 \\
B NaCl & 0.97 & 1.63 & 4.91 \\
C water & 0.96 & 1.85 & 4.56 \\
C NaCl & 0.97 & 1.22 & 4.61 \\
D water & 0.99 & 2.01 & 3.95 \\
D NaCl & 0.96 & 1.70 & 4.96 \\
E water & 0.97 & 2.09 & 4.04 \\
E NaCl & 0.97 & 1.99 & 4.77 \\
F water & 0.97 & 2.11 & 4.31 \\
F NaCl & 0.91 & 1.40 & 4.99 \\
G Water & 0.98 & 1.81 & 4.07 \\
G NaCl & 0.93 & 1.58 & 5.25 \\
\hline
\end{tabular}

The respective uncertainties are provided by the variance of the posterior distribution (see Fig. $2 \mathrm{~b}$ for the uncertainty in parameter $\mathrm{C}$ )

\section{Conclusion}

Proper data analysis is fundamental task of general interest in life sciences. Despite the current replication crisis and the dawning of "post $p<0.05$ era" $[2,3]$, this task is still not reflected enough in plant biology. Here, we present a novel and reliable method for the analysis of timeto-event data which is applicable to many developmental parameters measured in field or indoor conditions, e.g. seed germination, seedling emergence, the appearance of the first leaf, heading, flowering, fruit ripening, wilting, or death. In contrast to recent and classical approaches, our Bayesian computational method properly handles uncertainty in time-to-event data and it is capable to reliably answer questions that are difficult to address by classical methods.

\section{Methods}

Bayesian inference is general purpose method for handling uncertainty in experimental datasets. It should replace insufficient or biased "classical" statistical methods to provide more reliable data for better evidenced conclusions. Here we present an algorithm allowing such statistical analysis for time-to-event-data. The computational method is described above, whereas here we provide information about design of seedling emergence experiment that served as source of time-to-event data. However, this is only example of time-related dataset,
Bayesian survival analysis can be performed on similar data-type coming from very different conditions (field or indoor) or different developmental stage of studied plant population. The data were obtained from automated screening of maize emergence. Seeds of the maize (Zea mays L.) hybrid Koblens (KWS Osiva s.r.o., Czech Republic) were sown into TEKU JP 3050/160T nursery trays. Each tray was watered to the full water capacity with tap water or a solution of $150 \mathrm{mM} \mathrm{NaCl}$. Afterwards, all the trays were watered using $0.5 \mathrm{l}$ of tap water every third day until the end of the experiment. The trays were randomly assigned to the control or the salt stress groups at the beginning of the experiment.

Together with the salt stress, a second effector was introduced into the experiment. The seeds were primed either with water (control) or one of seven potential stimulatory compounds (compounds A-G). The treatment was applied during the imbibition phase by soaking the seeds in the respective solution for $16 \mathrm{~h}$ before sowing. Each treatment was evaluated in control and in salt stress conditions. Thus 16 trays were evaluated, each containing 110 seeds. The trays were placed into a controlled condition chamber with a robotically driven arm holding an RGB camera [17]. Each tray was photographed every $2 \mathrm{~h}$ for 12 days. The images were stored in a database server and analyzed semi-automatically by an in-house image processing routine. For each maize seed, the time of its emergence (i.e., the moment of the appearance of the coleoptile above the soil) was recorded.

\section{Supplementary information}

Supplementary information accompanies this paper at https://doi. org/10.1186/s13007-020-0554-1.

Additional file 1.1. Inadequacy of fitting of growth curves.; 2. Software

Acknowledgments

The work was supported from the ERDF project "Plants as a tool for sustainable global development" (No. CZ.02.1.01/0.0/0.0/16_019/0000827). Authors thanks to Abradatas company for development of free online tool based on our mathematical model.

\section{Authors' contributions}

JFH initiated a topic, tested and optimized mathematical model and participate in article writing. JD programmed mathematical model, generate visualization of data and was responsible for development of web page using our algorithm. LU prepared and performed seedling emergence experiment. NDD designed seedling emergence experiment and discussed the manuscript. LS discussed the manuscript and was responsible for funding of the work. OV participate in important part of model development. TF developed the model and wrote the mathematical part of the article. All authors read and approved the final manuscript.

\section{Funding}

The work was supported from the ERDF project "Plants as a tool for sustainable global development" (No. CZ.02.1.01/0.0/0.0/16_019/0000827). 


\section{Availability of data and materials}

The datasets during and/or analyzed during the current study available from the corresponding author on reasonable request. As we present here data analysis method, the sample data are free available to test in web http://www. bayes4plants.com. The code for the analysis is freely available here.

\section{Ethics approval and consent to participate}

Not applicable.

\section{Consent for publication}

Not applicable.

\section{Competing interests}

The authors declare the following potential competing interests: "In the manuscript, we provide a link to the webpage (bayes4plants.com) that serves as a free online tool for time-to-event data analysis in plant biology. One of the authors (JD) holds a stake in the company Abradatas that developed this webpage. The company developed the webpage free of charge. Although there is no direct financial gain for the company from the webpage, we declare this as a potential competing interest. Palacky University provided a consent to include this link in the manuscript to help the readers understand the Bayesian methodology of time-to-event data analysis in plant biology."

\section{Author details}

${ }^{1}$ Department of Chemical Biology and Genetics, Centre of the Region Haná for Biotechnological and Agricultural Research, Faculty of Science, Palacký University, Šlechtitelů 27, 78371 Olomouc, Czech Republic. ${ }^{2}$ Department of Mathematical Analysis and Application of Mathematics, Faculty of Science, Palacký University, 17. listopadu 12, 77900 Olomouc, Czech Republic

Received: 6 November 2019 Accepted: 16 January 2020

Published online: 11 February 2020

\section{References}

1. Araus JL, Cairns JE. Field high-throughput phenotyping: the new crop breeding frontier. Trends Plant Sci. 2014;19:52-61.

2. Goodman SN. How sure are you of your result? Put a number on it. Nature. 2018:564:7-7.

3. Matthews RAJ. Beyond 'significance': principles and practice of the analysis of credibility. R Soc Open Sci. 2018:5:171047.
4. Ranal MA, de Santana DG. How and why to measure the germination process? Rev Bras Botânica. 2006;29:1-11.

5. Joosen RVL, et al. Germinator: A software package for high-throughput scoring and curve fitting of Arabidopsis seed germination. Plant J. 2010:62:148-59.

6. Archontoulis SV, Miguez FE. Nonlinear regression models and applications in agricultural research. Agron J. 2015:107:786-98.

7. McNair JN, Sunkara A, Frobish D. How to analyse seed germination data using statistical time-to-event analysis: non-parametric and semi-parametric methods. Seed Sci Res. 2012;22:77-95.

8. Hay FR, Mead A, Bloomberg M. Modelling seed germination in response to continuous variables: use and limitations of probit analysis and alternative approaches. Seed Sci Res. 2014;24:165-86.

9. Onofri A, Mesgaran MB, Neve P, Cousens RD. Experimental design and parameter estimation for threshold models in seed germination. Weed Res. 2014;54:425-35.

10. Onofri A, Piepho H-P, Kozak M. Analysing censored data in agricultural research: a review with examples and software tips. Ann Appl Biol. 2019;174:3-13

11. Kleinbaum DG, Klein M. Introduction to survival analysis. New York: Springer; 2012. https://doi.org/10.1007/978-1-4419-6646-9

12. Klein JP, Moeschberger ML. Survival analysis techniques for censored and truncated data. New York: Springer; 2003. https://doi.org/10.1007/b97377

13. Hosmer DW, Lemeshow S, May S. Applied survival analysis : regression modeling of time-to-event data. Hoboken: Wiley-Interscience; 2008

14. Austin PC. Statistical power to detect violation of the proportional hazards assumption when using the Cox regression model. J Stat Comput Simul. 2018;88:533-52.

15. Hoffman MD, Gelman A. The No-U-Turn sampler: adaptively setting path lengths in hamiltonian monte carlo. J Mach Learn Res. 2014;15:1593-623.

16. Salvatier J, Wiecki TV, Fonnesbeck C. Probabilistic programming in Python using PyMC3. PeerJ Comput Sci. 2016;2:e55.

17. De Diego N, Fürst T, Humplík JF, Ugena L, Podlešáková K, Spíchal L. An automated method for high-throughput screening of Arabidopsis rosette growth in multi-well plates and its validation in stress conditions. Front Plant Sci. 2017:8:1702

\section{Publisher's Note}

Springer Nature remains neutral with regard to jurisdictional claims in published maps and institutional affiliations.
Ready to submit your research? Choose BMC and benefit from:

- fast, convenient online submission

- thorough peer review by experienced researchers in your field

- rapid publication on acceptance

- support for research data, including large and complex data types

- gold Open Access which fosters wider collaboration and increased citations

- maximum visibility for your research: over 100M website views per year

At BMC, research is always in progress.

Learn more biomedcentral.com/submissions 